\title{
Evaluation of wind-driven rain exposure to buildings in China
}

\author{
Huibo Zhang ${ }^{\text {a, *, Tianda Qian }}{ }^{\text {a }}$ \\ a Department of Architecture, School of Design, Shanghai Jiao Tong University, Shanghai, China
}

\begin{abstract}
To promote China's sustainable building technology, it is necessary to conduct an overall evaluation of the building lifetime exposure to winddriven rain (WDR). The annual driving rain index (aDRI) and airfield absolute spell index ( $\left.I_{\mathrm{AS}}\right)$ values of 141 representative areas were calculated by using the meteorological data of past 30 years. Then based on ArcGIS 10.4, the long-term and extreme exposure maps of WDR in various China regions were drawn. The results show that extreme and long-term WDR exposure is particularly severe in South China's coastal areas, which require particular attention. The exposure to WDR is gradually decreasing from the coast to the inland regions.

Peer-review under the responsibility of the organizing committee of the ICMB21.
\end{abstract}

Keywords: China; wind-driven rain (WDR); ArcGIS; long-term exposure; extreme exposure;

\section{Introduction/Background}

The primary moisture source in building components is wind-driven rain (WDR), impacting the exterior wall materials and buildings' thermal and moisture properties. Studying the exposure of building components to WDR is of great significance for estimating the expected wet load and taking appropriate protective measures. The main methods for estimating the WDR amount of building walls include field measurement, numerical simulation, and semi-empirical methods. The semi-empirical method's main advantage is that it is simple to calculate and quickly assess WDR exposure, conducive to practical engineering applications.

In some past studies, different types of semi-empirical formulas have been derived to qualitatively assess WDR exposure, such as the driving rain index (DRI), calculated from horizontal rainfall and wind speed during rains. DRI calculated based on the meteorological data within a year is called the annual driving rain index (aDRI). The index is proportional to the amount of water on the building's windward surface [1]. The aDRI calculated from daily, monthly, and annual average meteorological data is called daDRI, maDRI, and aaDRI. According to calculated daDRI scores, Lacy and Shellard [1] divided each region's exposure situation into shield, moderate, high, and severe classification. In recent years, some countries have conducted driving rain exposure assessments and drawn driving rain maps based on this, such as Spain [2], Greece [3], Brazil [4], and India [5].

China has a vast territory, complex terrain, large climate differences between regions, and the world's most existing buildings. In order to promote the development of sustainable building technology and the maintenance of existing buildings in China, it is urgent to evaluate the current WDR exposure of building exterior walls in different regions of China and draw China's WDR map accordingly to depict the WDR exposure.

\section{Evaluation methods}

This paper comprehensively considers the meteorological characteristics, administrative divisions, longitude, and latitude span of each region in China to evaluate the current wind-driven rain exposure in various China regions, then 141 municipal regions are selected as representatives. The meteorological data of meteorological stations in these regions are obtained through China Integrated Meteorological Information Service System (CIMISS), including daily average wind speed (m/s) and daily rainfall (mm), with a period of 30 years (1990-2019). Based on the daily rainfall (mm) and the average wind speed $(\mathrm{m} / \mathrm{s})$ of each day in every year, the daDRI of each region is calculated, as shown in formula (1). Based on daily meteorological data, $I_{A S}^{\prime}$ is used to describe the characteristics of building components in all directions at the location exposed to WDR in a particular wet stage with a return period of 3 years. The calculation formula is shown in formula (2).

$$
d a D R I=\frac{1}{n} \times \sum_{i=1}^{k}\left(h_{i}^{d} \times u_{i}^{d}\right)
$$

$$
I_{A S}^{\prime}=\frac{2}{9} \sum U_{10} \cdot\left(R_{h}\right)^{8 / 9}
$$

In which, $\mathrm{n}$ is the number of years of meteorological data obtained, which is taken as 30 ; $k$ is the total number of days in these years, $h_{i}^{d}$ is the rainfall of day $i(i=1,2,3 \mathrm{k})(\mathrm{mm}), u_{i}^{d}$ is the daily average wind speed on day $i(i=1,2,3 \mathrm{k})(\mathrm{m} / \mathrm{s}) ; 2 / 9(\mathrm{~s} / \mathrm{m})$ is the WDR coefficient determined according to the raindrops' falling speed. $U_{10}(\mathrm{~m} / \mathrm{s})$ is the wind speed at the unobstructed reference height of 10 meters in the absolute spell; $R_{h}(\mathrm{~mm} / \mathrm{h})$ is the total rainfall in the absolute spell.

\footnotetext{
* Corresponding author: Huibo Zhang, +86 13818784711, zhanghuibo@situ.edu.cn.
} 
ICMB21

\section{Results}

\subsection{Long-term exposure}

The WDR exposure levels in China are divided into six detailed levels, values from low to high are shown as: "shielded", "low", "moderate", "high", "very high", "severe". Based on this classification rule, the long-term WDR exposure map of different regions in China (based on the CGCS_2000 plane coordinate system) is shown in Fig. 1a. Long-term exposure to WDR is the most serious in the coastal provinces of South China, such as Guangdong, Hong Kong, and Macao, the value is even higher than 6, belonging to the "severe" level. Secondly, most of Guangdong, Hainan, Guangxi, and some parts of Zhejiang Province are also seriously exposed to WDR, reaching the "very high" level. Jiangsu, Zhejiang, Fujian, Taiwan, Jiangxi, and other provinces are affected by "high" WDR. Also, in Central China, the long-term exposure level of WDR is "moderate" or "low", which is affected by light WDR. Inland provinces such as Xinjiang, Gansu, Qinghai, and Ningxia are almost not affected by WDR exposure, and their daDRI is mostly lower than 1 , at the "shielded" exposure level.

\subsection{Extreme exposure}

Based on above classification rule, the extreme WDR exposure maps of various China regions are obtained after interpolation, as shown in Fig. 1b. In South China coast: Guangdong, Hainan, and parts of Guangxi have the most "severe" extreme WDR exposure, and the coastal areas of Shandong, Jiangsu, Zhejiang, and Fujian provinces and most parts of Yunnan have "high" extreme WDR exposure. Compared with Fig. 1a, except for some specific areas, such as Yunnan Province, areas with severe longterm exposure levels (with higher daDRI values) also bear more severe extreme WDR loads during the wetting period.

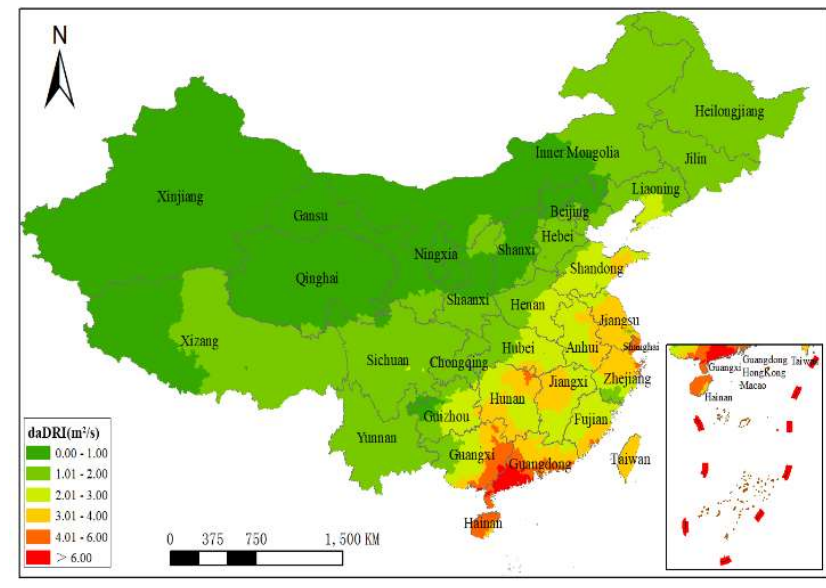

(a)

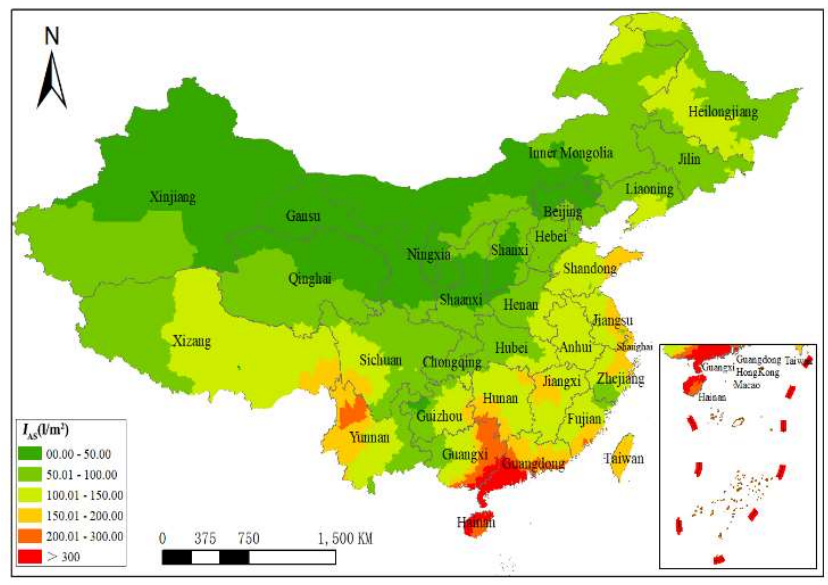

(b)

Figure 1. (a) Long-term exposure map of WDR in various regions of China; (b) Extreme exposure map of WDR in various regions of China

\section{Conclusions}

The daDRI values of Guangdong, Hainan, and parts of Guangxi in southern China are mostly higher than $4 \mathrm{~m}^{2} / \mathrm{s}$, and $I_{A S}$ values are mostly higher than $2001 / \mathrm{m}^{2}$, which proves that their long-term and extreme exposures are relatively severe and should be paid attention to when formulating corresponding building codes. The long-term and extreme WDR exposure changes in the regions are similar. From the southern and southeast coastal areas, the WDR exposure gradually decreases toward the inland areas.

\section{Acknowledgement}

This work is financially supported by the National Natural Science Foundation of China (No. 51778358).

\section{References}

[1] Lacy R.E., Shellard H.C. (1962). An index of driving rain. Meteorol. Mag 91:177-184.

[2] Perez-Bella J.M., Dominguez-Hernandez J., Rodriguez-Soria B., Coz-Diaz J.J.D., Cano-Sunen E. (2012). Estimation of the exposure of buildings to driving rain in Spain from daily wind and rain data. Building \& Environment 57(11): 259-270.

[3] Giarma C., Aravantinos D. (2014). On building components' exposure to driving rain in Greece. J. Wind Eng. Ind. Aerodyn. 125:133-145.

[4] Dominguez-Hernandez J., Perez-Bella J.M., Alonso-Martinez M., Cano-Sunen E., Coz-Diaz J.J.D. (2017). Assessment of water penetration risk in building facades throughout Brazil. Building Research and Information 45(5): 492-507.

[5] Narula P., Sarkar K., Azad S. (2018). Indexing of driving rain exposure in India based on daily gridded data. J. Wind Eng. Ind. Aerodyn. 175: 244251. 